\title{
The Future Laboratory: Leveraging Consumer Imaging Devices for Student Projects and Sustainable, Accessible STEM Education.
}

\section{Dr. Michael G. Mauk, Drexel University}

Michael Mauk is Assistant Professor in Drexel University's Engineering Technology program.

\section{Dr. Richard Chiou, Drexel University}

Dr. Richard Chiou is Associate Professor within the Engineering Technology Department at Drexel University, Philadelphia, USA. He received his Ph.D. degree in the G.W. Woodruff School of Mechanical Engineering at Georgia Institute of Technology. His educational background is in manufacturing with an emphasis on mechatronics. In addition to his many years of industrial experience, he has taught many different engineering and technology courses at undergraduate and graduate levels. His tremendous research experience in manufacturing includes environmentally conscious manufacturing, Internet based robotics, and Web based quality. In the past years, he has been involved in sustainable manufacturing for maximizing energy and material recovery while minimizing environmental impact.

\section{Prof. Tzu-Liang Bill Tseng, University of Texas, El Paso}

Dr. Tseng is a Professor and Chair of Industrial, Manufacturing and Systems Engineering at UTEP. His research focuses on the computational intelligence, data mining, bio- informatics and advanced manufacturing. Dr. Tseng published in many refereed journals such as IEEE Transactions, IIE Transaction, Journal of Manufacturing Systems and others. He has been serving as a principle investigator of many research projects, funded by NSF, NASA, DoEd, KSEF and LMC. He is currently serving as an editor of Journal of Computer Standards \& Interfaces. 


\section{The Future Laboratory: Leveraging Consumer Imaging Devices for Student Projects and Sustainable, Accessible STEM Education}

\section{Introduction}

Industry, healthcare and STEM education have often relegated chemical analysis, surface characterization, bioassays, and measurements that require special types of sensors (e.g., $\mathrm{pH}$ or fluorescence) to laboratories or venues with comparatively expensive and sophisticated equipment, skilled technicians, and well-controlled conditions. Recent technology trends seek to develop minimally-instrumented, portable (handheld) systems that can perform chemical and biochemical analysis and characterization of samples outside of laboratory settings, and devices that can provide a more global analysis of sample features such as surface condition, cleanliness, and defects. Digital imaging (visible, infrared, and fluorescence) is set to transform medical diagnostics, especially when connected to the Internet of Medical Things, a network of sensors, medical devices, healthcare providers, and data bases, as well as other fields such as environmental monitoring, quality assurance, energy efficiency, food safety, and homeland security. Therefore, there is much incentive to integrate these new digital imaging technologies in modes and formats to gain quantitative data such as chemical detection in the engineering curricula, and also to leverage their affordability and power for sustainable STEM education. This paper provides a survey of various imaging modalities, especially those suitable for undergraduate laboratories and projects, that have been developed over the past decade. Much of this work has been reported in the chemistry education literature, and may not have gained sufficient attention for engineering education, or may sometimes be dismissed by engineering instructors as primarily of interest to chemistry and biology students rather than electrical, mechanical, and industrial engineering students. Imaging, including fluorescence, luminescence, optical absorption, and infrared thermal imaging, as well as videos and image processing and analysis can add new dimensions to engineering measurement-based laboratories. We also review applications of imaging technology for engineering topics that we have developed over the last five years, including defect analysis of solar cells, and mixing and heat transfer in microscale system. Many of these methods use low cost digital cameras, smartphone cameras, and readily-available image processing software, thus offering sustainable, low-cost instrumentation that can be widely disseminated to educational institutions with limited resources.

Many analytical techniques can be implemented with imaging and optical detection devices such as smartphones, low-cost digital cameras and USB 'microscopes', desktop scanners, and modified CD players. For example, the CCD camera of a smartphone can be used as an optical detector in absorption, reflection, scattering, and fluorescence measurements, albeit for some methods requiring also an optical source (e.g., and LED) and optical filters. Color cameras can discriminate wavelengths, thus allowing spectroscopic measurements. These pervasive technologies are highly familiar and accessible to students, and offer additional features such as connectivity, data processing and archiving, GPS, cloud computing, and virtual reality. There are many reports spread through research literature in adapting such inexpensive, ubiquitous 
consumer devices for optical analysis (absorption, fluorescence, luminescence, colorimetry), thermal (infrared) imaging, product inspection, remote sensing, environmental monitoring, bioassays, and various medical tests. Digital imaging can also be used to assess surface roughness, surface cleanliness, and wettability (contact angles). Thermal imaging with low-cost infrared cameras can be used to analyze defects in electronic devices such as solar cells, and thermal and fluid flow patterns in microsystems. In this paper, we will survey some representative applications of special interest to engineering education, including methodologies we have developed microfluidics, solar cells testing, surface roughness measurements and POC medical diagnostics.

\section{Imaging Technologies Are Opening New Vistas for Measurement Science}

Microscopy and photography have been important tools of science, technology, and medicine for most of the modern era. Digital imaging, as enabled by CCD cameras, scanners, and thermal detector arrays and other image capture devices has revolutionized measurement and analysis due to its low-cost, convenience, and accessibility, high resolution and extension to previously unexplored parts of the electromagnetic spectrum, and digital format which facilitates storage, processing, and analysis.

It is estimated that by the year 2020, there will be 2.9 billion smartphones in the world [1]. OzCAN [2] provides a high-level overview of mobile phones for imaging/microscopy, sensing, medical diagnostics and general measurement science, enabled by the pervasiveness, low cost, connectivity, and increasing performance of mobile phones with CCD cameras and other accessories. A few trends noted by OzcAn [2] are analogous to Moore’s Law in microelectronics: 1 . The yearly increase in pixel count of mobile phone images is exponential, 2. computer processing power of mobile phones is also increasing exponentially, approaching that of PCs, 3. Mobile phone network speed is increasing and closed to the average speed of internet communication rates (bps), and 4. The cost of data transmission (\$/Mbit) via mobile phone continues to decline, e.g., by more than a factor of 10 between the years 2008 and 2012. The developments suggest ever wider applications and performance can be expected in the next decade. OzCAN [2] predicts that mobile phones will foster a transformation that will 'democratize' measurement science and practice worldwide, which “might significantly improve research and education... especially in developing countries”. Biomedical and biosensing applications (including accessories for electrochemical detection, and sensors for magnetoresistance, sound recording, in addition to optical detection) with mobile phones is reviewed by QUESADA-GONZALEZ and MERKOÇI [3]. This review featured many illustrations of 3-printed and rapid prototyped plastic parts to implement analysis techniques with the cellphone. WALKER et al. [4] have described efforts to use mobile phones as genetic diagnostics systems, integrating DNA chemistry technology with cellphone camera detectors.

Even simple imaging experiments will give students useful experience with the technologies and methods for image capture, processing, and analysis. Image processing is currently a specialized area of electrical engineering, and is used often in experimental engineering for microscopy. Image analysis can validate two- and three-dimensional multiphysics modeling (finite element and finite difference maps of temperature, stress, fluid flow, electric fields) that 
students increasingly used in their CAD work. SCOTT and MCCANN [5] stress the increasing importance of imaging of chemical, manufacturing, energy extraction and conversion processes, as made feasible by high-performance imaging systems, for process control and optimization. Below, we introduce and describe these using thermal cameras and microfluidic systems.

\section{A Survey of Imaging in STEM Education}

Many of the experiments described below can be done as well (and perhaps better) with conventional laboratory equipment such as microscopes, spectrometers, dedicated CCD and thermal cameras, however, the use of portable or desktop devices brings students closer to the measurement process, allows use outside of laboratories, e.g., for environmental monitoring or field engineering projects, and will no doubt produce a dividend in cost savings, as alreadyowned devices by students or schools can be repurposed for double-duty as measurement tools. Furthermore, the connectivity of such devices facilitates sharing of data for collaborative work, including between schools.

\section{Smartphones and Digital Cameras}

Mobile phones with CCD cameras ('smartphones) and other consumer-grade digital cameras are proving versatile platforms for healthcare, environmental monitoring, portable analytical instruments, and STEM education. MCGONIGLE et al. [6] note that in 2017, there were over 250 publications on Smartphone imaging.

CAMPOS et al. [7] took cellphone camera images of suspensions of gold nanoparticles in aqueous solutions at various concentrations in clear plastic cuvettes. Using a free web-based 'color picker' software, images were resolved into Red-Green-Blue levels, such that colorimetry calibration curves could be constructed for known serial dilutions of gold nanoparticles, thus providing for determination of the concentration of unknown samples. SHARPE and ANDREESCU [8] reported a similar approach where the color change of nanoparticles adsorbed on paper strips in order to measure antioxidant compounds in food. As a corollary benefit, such experiments give students exposure to nanotechnology. Tests strips (e.g., $\mathrm{pH}$ paper, and lateral flow strips) are a well-developed analysis method. Such paper-based methods use capillary effects in porous materials such as nitrocellulose to wick samples and reagents through capture zones where they bind and are decorated with reporters such as fluorescent compounds are nanoparticles. Such test strips are now sold over the counter in pharmacies and include home pregnancy tests, drugs of abuse tests, and tests for HIV using saliva. The reporters can be visualized by eye, but improved sensitivity, multiplexing, and semi-quantitative assays can be realized by using an optical detector such as a Smartphone camera [9, 10]. Lu et al. [11] used a cellphone camera for low-cost, portable detection of gold nanoparticle-labeled microfluidic immunoassays.

\section{Smartphone-Based Microscopes}

Smartphones can be adapted as microscopes. They are considerably cheaper than conventional microscopes, and are a device students that are very familiar to students. Crystal growth in particular, and solidification in general are important phenomena in materials science. LUMETTA and ARCIA [12] used a smartphone-based microscope to observe dissolution and precipitation in 
crystallizing sodium chloride $(\mathrm{NaCl})$ from aqueous solutions. The Smartphone microscope used a glass bead mounted in a 3d printed to give a 100x magnification and a depth of field suitable for observing objects in the size range of microscope slides. Also, mobile phone microlens attachments for cellphones (giving 60X magnification) are available for a few dollars.

\section{Smartphone Spectrometers}

Custom-made Smartphone-based spectrometers have been described in the literature [6]. Spectrometers as accessories to Smartphones have been developed that have resolution (10 nm) comparable to benchtop instruments [13]. Commercially-available spectrometer units that attach directly to Smartphones as an accessory are currently available for (\$1200 to \$2000). Based on historic trends of microelectronics and CCD cameras, it is plausible that the prices of such spectrometers may be considerably reduced in the coming decade.

\section{Desktop Scanners}

Desktop flatbed scanners are a convenient means to image flat, thin samples such as sheets, silicon wafers and solar cells, filter paper, fabrics, test strips, microfluidic chips, microtiter plates, and Petri dishes. They comparatively inexpensive (\$200 to \$500), have a large imaging field of view (600 to $700 \mathrm{~cm}^{2}$ - A4 or $81 / 2$ x 11 inch paper size), but a modest spatial resolution (10 microns) [14]. The large image size stems from the linear scanner mode, rather than relying on a large-pixel CCD camera. Scanners that use a CCD type imaging system (rather than a CIS, Contact Image Sensor) have a depth of field of 1 to $2 \mathrm{~mm}$, allowing imaging of thicker objects such as Petri dishes. The illumination is typically provided by red, green, and blue LEDs, but in some cases by a fluorescent lamp. Image sizes may be 100s of Megabytes. They have been used in colorimetric assays (qualitative chemical identification based on reflectance spectra); optical absorption based assays, particularly for imaging metal nanoparticles, microscopy of various biological specimens, e.g., tissue sections; and in fluorescence imaging detection, as well as some more specific examples discussed below. SHISHKIN et al. [15] discussed the use of desktop scanners for quantitative analysis of various compounds (e.g., transition metals, nitrites, inorganic acids) with wavelengths of maximum diffuse reflectivity in the range of 380 to $620 \mathrm{~nm}$ adsorbed on polyurethane foam. (Non-linear, first-order exponential) calibration curves for all three colors (red, blue, green) of a single pixel of a color image were made demonstrating sensitivity limits as low as $0.01 \mu \mathrm{g} / \mathrm{ml}$.

ZHELEZNYAK and SIDOROV [16] used a flatbed scanner to measure scattered light from samples in order to estimate surface roughness (down to $2 \mathrm{~nm}$ rms roughness, with 3 to $10 \mu \mathrm{m}$ lateral resolution), scratches and defect densities, including their position, shape, and size; and bowing or surface curvature, of various metal, glass, semiconductor, and dielectric specimens.

SolDAT et al. [17] described the use of a desktop scanner and digital image analysis software for microscale colorimetric analysis. They placed a standard 96-well microplate in a commercial desktop scanner (Epson, 200 dot-per-inch resolution, cost $~ \$ 400$ ) operating in the transparency mode. Scanned images were analyzed with public-domain image analysis software (ImageJ) allowing co-ordinate analysis ( $x, y$ pixel position) of the image areas corresponding to a sample in a microwell. The mean, median, and mode values of the red, green, and blue channels for each 
pixel are determined. Calibration curves for each color are then made for serial dilutions of phosphate, ammonia, nitrates solutions enabling analysis of samples in the $\mathrm{mg} / \mathrm{L}$ range. A suggested application is a battery operation of scanner as a portable instrument for remote or field environmental monitoring.

PocE-FATOU et al. [18] used a desktop flatbed scanner as a reflectance spectrometer. The scanned image of fabrics soiled with oils and then immersed in beakers of magnetically-stirred detergent solutions could be used to assess the efficacy of detergents in removing oil from fabrics and restoring their original color. MATHEws et al. [19] applied similar approaches to adapt a desktop scanner to measure the starch level in food samples (mixed with iodine as a color indicator of starch).

OsKOLOK et al. [20] described improvements through modifications and accessory components, including sample holders, a laser light source and optical filters, to office flatbed scanners to improve their use in colorimetry, photometry, fluorimetry, and nephelometry (light scattering of liquid suspensions). Similar endeavors would serve as useful educational projects for engineering students. KEARNS and TYSON [21] developed an arsenic field test unit based on digital analysis of test strip images captured with a desktop scanner.

MAUK et al. [22] used a desktop scanners to image the grain structure of silicon solar cells. Standard image processing software could be used to evaluate grain size distribution and grain texture, which impact the performance of solar cells, see Figures $\mathbf{1}$ and $\mathbf{2}$. Similar type analysis in metallography (with etched metal samples) is common in materials science educational laboratories using optical microscopes. Also, solar cell defects such as broken grid lines or scratches can be detected $[23,24]$. The use of image processing for determining surface roughness of specimens serves as an accessible introduction to extracting information from images readily captured with a digital camera. One relatively simple procedure is to analyze grey-level histograms of pixel intensity distributions of images of surfaces [25]. Histogram distribution parameters can be correlated with surface roughness. More sophisticated image processing for surface roughness analysis of various sample types, including solar cells, machined surfaces, and woven materials has been reported [26,27]

Two other types of educational experiments are enabled or streamlined by digital cameras. Wetting experiments measure the wetting angle of liquid drops on a surface. An image of the cross section of the drop can be digitized to determine wetting angles for different types of surfaces and liquids (hydrophilic vs hydrophobic) as well as the effects of contaminants and surfactants [28-29]. Wetting figures prominently in coatings, cleaning procedures, corrosion, adhesion, and lubrication. Handheld fluorescent digital microscopes, that plug into the USB port of computers include blue or UV LEDs to excite fluorescent compounds, and filters that allow fluorescent imaging. One interesting application is to evaluate the effectiveness of cleaning procedures as perhaps a demonstration of 'green' manufacturing. We contaminated metal and silicon wafer surfaces with organic fluorescent dyes, and then optimized cleaning methods (rinse volume, number of rinses, temperature of water, detergent concentration) and imaged the surface to determine residual fluorescence as an indicator of imperfect or incomplete cleaning. 

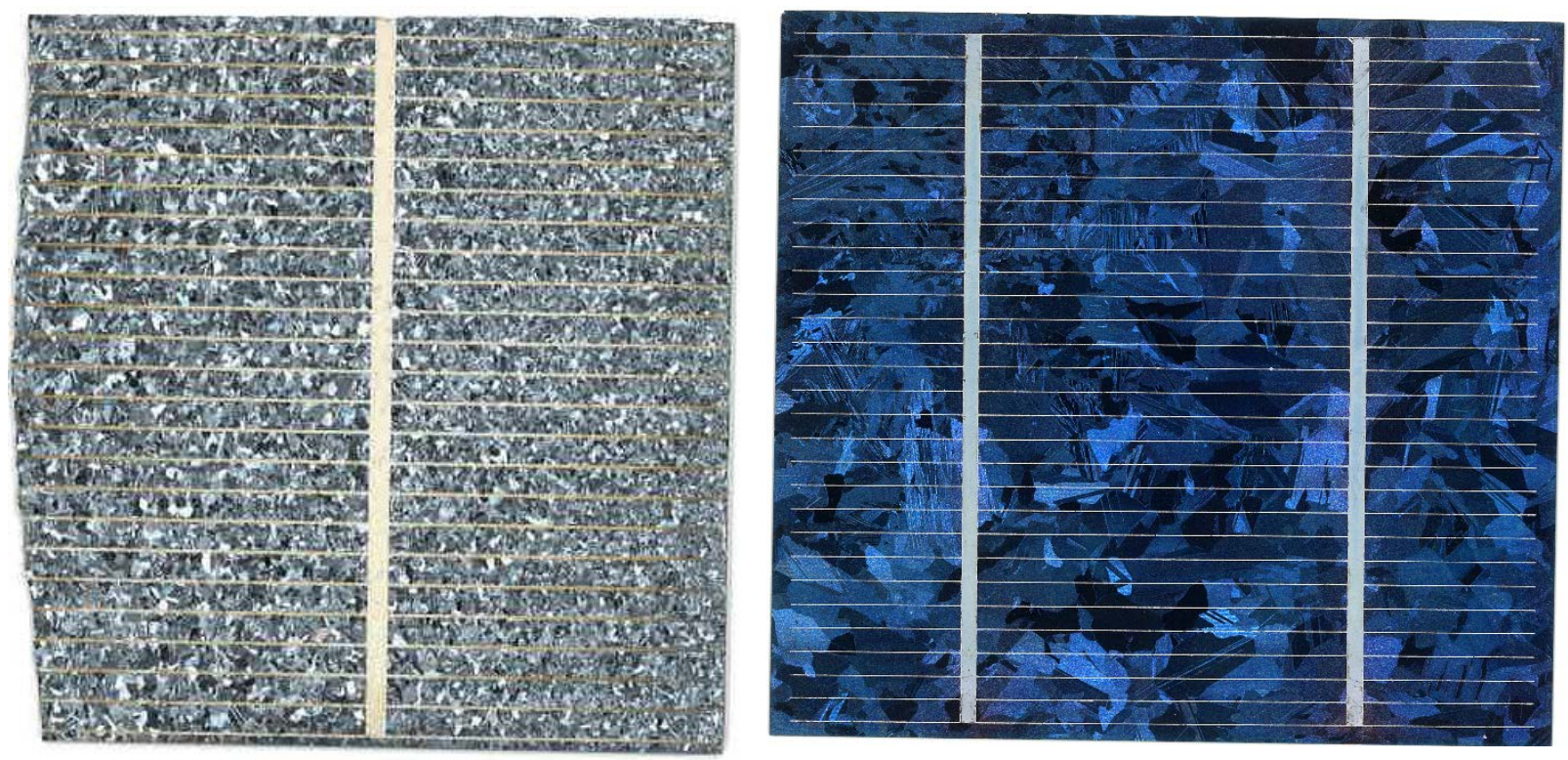

Figure 1: Polycrystalline silicon solar cells with screen printed aluminum contacts, without antireflection coating (left) and with $\mathrm{TiO} 2$ ('blue') anti-reflection coating (right) are imaged for grain structure and defect analysis. Cells are 5-cm x 5-cm.
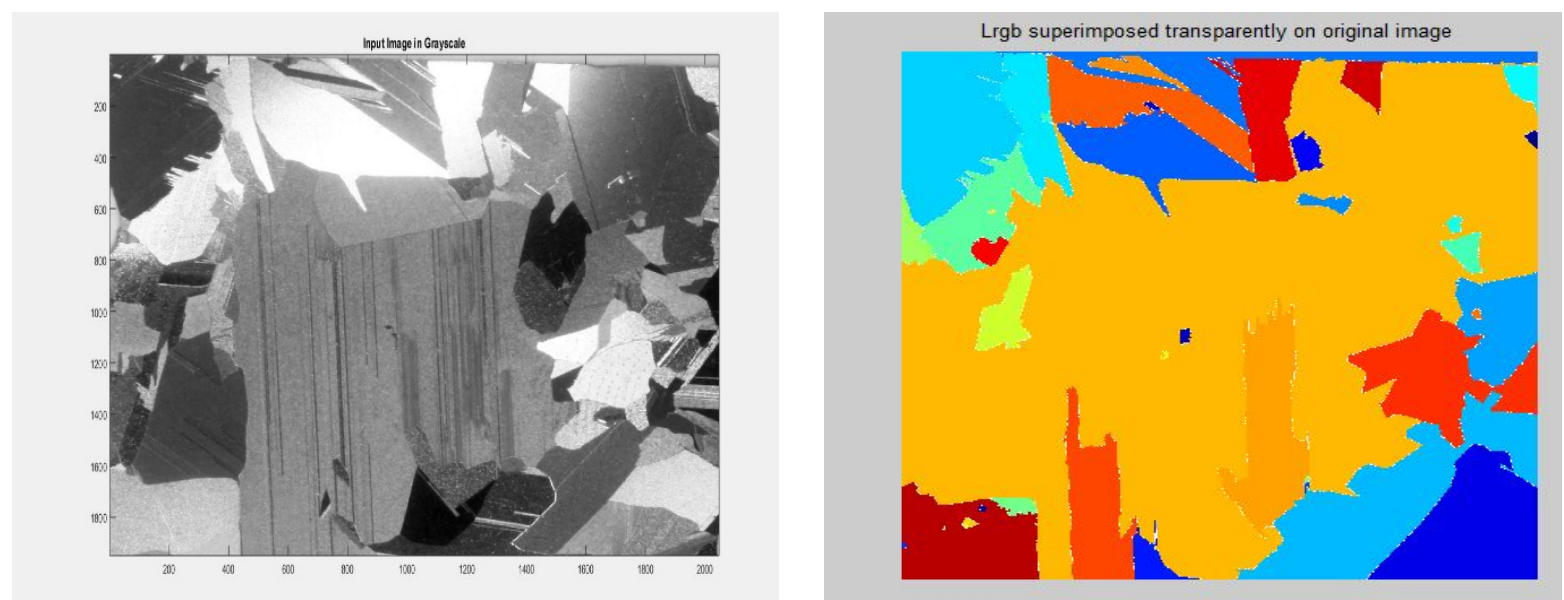

Figure 2: Polycrystalline silicon wafers imaged with a digital camera. Grey scale image on left, colorcoded image on right showing different grains and their orientation. The grain structure of silicon wafers has considerable impact on the performance and reliability of silicon solar cells made from such wafers.

Videos captured with CCD digital cameras can be used to analyze fluid flow in microfluidic chips (Figures 3 and 4). Plastic chips made in acrylic or polycarbonate sheet as bonded laminates are machined with a CO2 laser or CNC machine to define micro-scale ( $\sim \mathrm{mm})$ fluidic networks. Flow rate and mixing can be quantified by analysis of each successive video frame. Thermal cameras (see below) can 'image' temperature profiles and heat flow in such chips. These chips provide microscale versions of heat and mass transfer experiments, making only 
modest demands on laboratory space, consumables, waste disposal, and safety precautions, and thus are attractive as sustainable alternatives to more traditional engineering education laboratories.

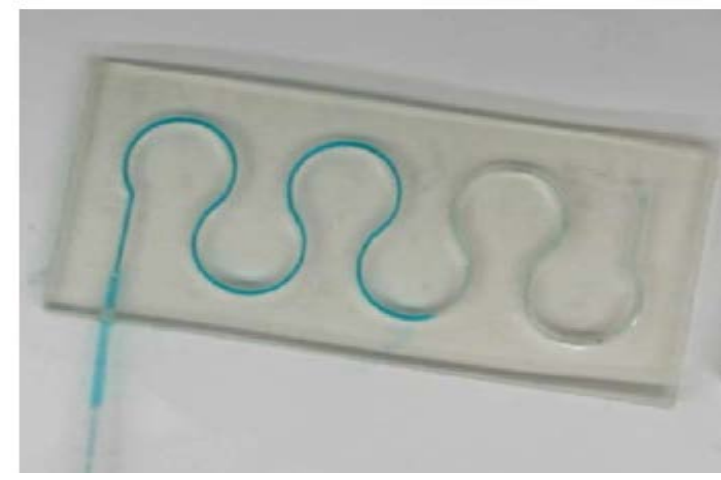

Figure 3: A dyed liquid is fills a serpentine microfluidic channel. The fluid flow is imaged with a CCD camera in the video mode. Each frame of the video is digitized, and (based on color differences) the flow front progression in each frame is determined.

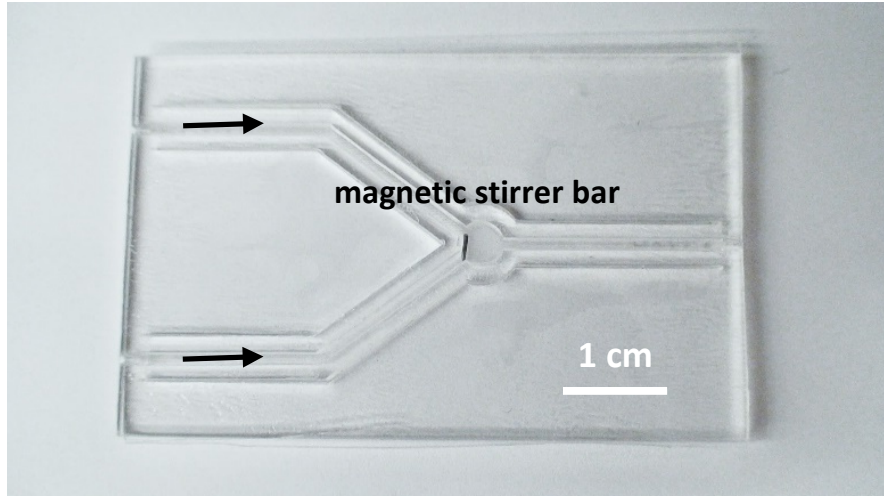

Figure 4: A microfluidic chip for mixing experiments. Two channels meet at a mixing chamber with a magnetic stirrer (actuated by a rotating magnet of a stirrer/hotplate platform. Different color dyes in each chamber can indicate the flow patterns and the degree of mixing.

\section{Inexpensive Infrared Cameras}

Infrared (8 to $14 \mu \mathrm{m}$ wavelength) thermal camera imaging (thermography) expands the applications of image analysis as they provide two-dimensional maps of the surface temperature of solids and fluids. Low-cost infrared cameras can resolve temperatures (with emissivity correction) to $\pm 0.5^{\circ} \mathrm{C}$. Many reports of the use of infrared cameras in STEM education emphasize the added visualization IR cameras provide to an area of science that previously was interpreted primarily through mathematical models. XIE [30-32] has surveyed and summarized the use of IR cameras for qualitative understanding in secondary and undergraduate STEM education and inquiry-based learning in visually exploring phenomena such as condensation and evaporation, convective flow in air and liquids, crystallization, phase transitions, heat transfer, infrared absorption, and capillary action. MöLlMAN and VOLLMER [34] discuss infrared thermal imaging in university physics education.

\section{Some Engineering Education Examples}

We have developed two thermal imaging experiments for undergraduate engineering: 1) thermal imaging of solar cells under bias to detect defects and hot spots, and 2) thermal imaging of exothermic reactions in microfluidic chips.

Figure 5 shows the set-up (5a) and a thermal image (5b) of a 5-cm x 5-cm silicon solar cell under forward voltage bias with a 1-Amp current. Hot spots are due to uneven distribution of current and defective regions that act as shunt paths, resulting in localized heating. The central metallized busbar (vertical hot region in center of line of solar cell) shows significant 
heating due to resistive losses. This demonstration is used by students in undergraduate renewable energy courses to understand and assess the role of various defects in semiconductor devices and their impact on performance.

Figure 6 shows a microfluidic chip (plastic cartridge) with a porous cellulose filter disk intercepting a flow channel. Green-dyed water is driven through the channel using a programmable syringe pump. The flow pattern of liquid through the porous material is readily revealed by time lapse images with a digital camera.

Figure 7 shows thermal image of a chip where a stream of sulfuric acid and a stream of $\mathrm{NaOH}$ are continually mixed, resulting in a product stream of elevated temperature. The prospects of doing microscale heat and mass transfer and fluid mechanics experiments on student-designed chips, and monitoring the results offers a sustainable, multidisciplinary option for bench-scale laboratory experiments typically attached to engineering courses in Mechanical, Electrical, and Chemical Engineering. We emphasize the considerable increase in data and information afforded by imaging of flow, as compared to that attained by using a small number of point sensors (e.g., thermocouple probes). Further, a dynamic picture of mixing and heat transfer is possible by video formats. These images are readily compared to finite element modeling of fluid flow and heat transfer.
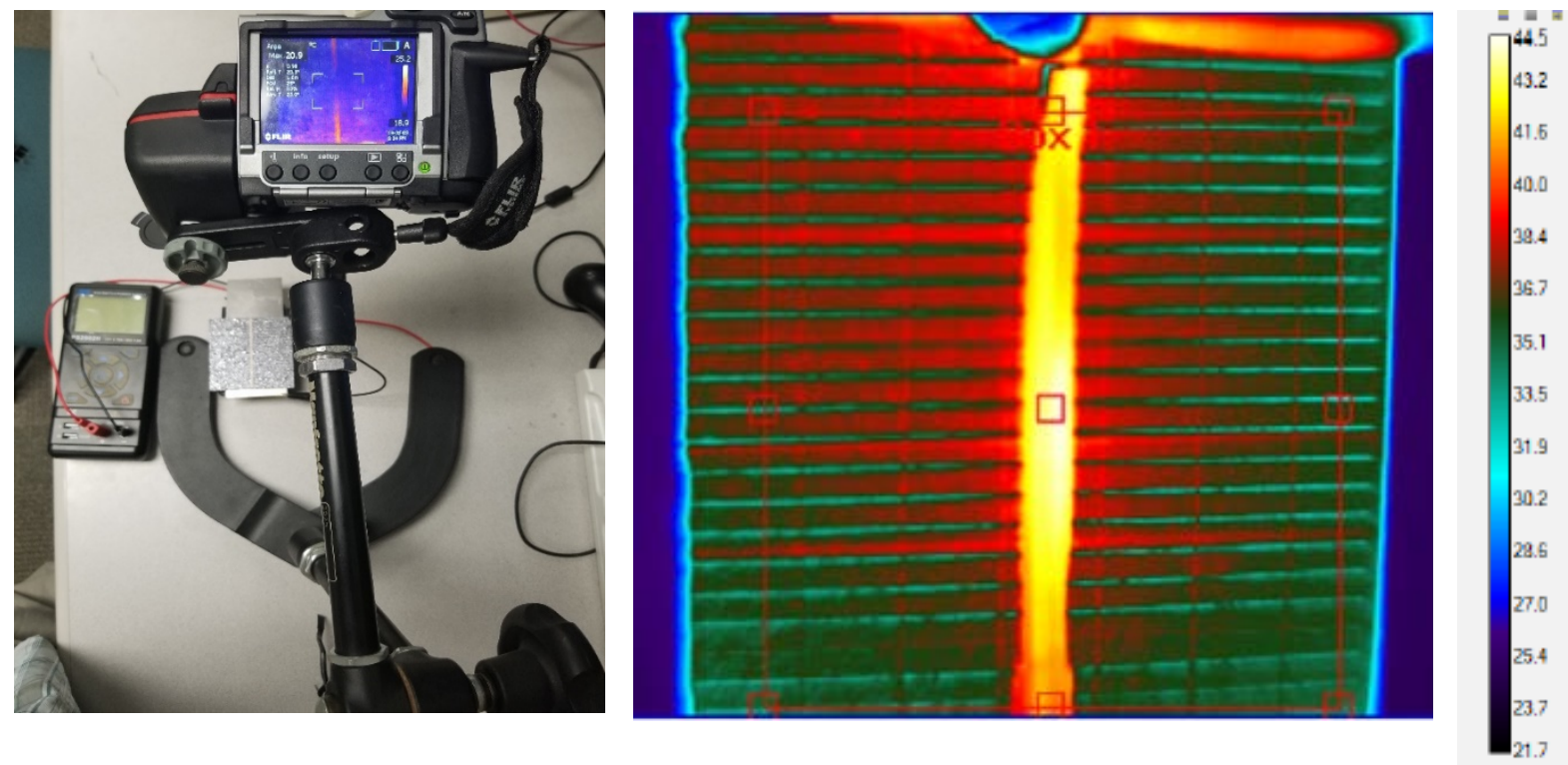

Figure 5: Left (a): Infrared camera for thermal imaging silicon solar cells. Right (b): Temperature profile of solar cell under forward bias. Red areas show localized heating due to shunt defects. 

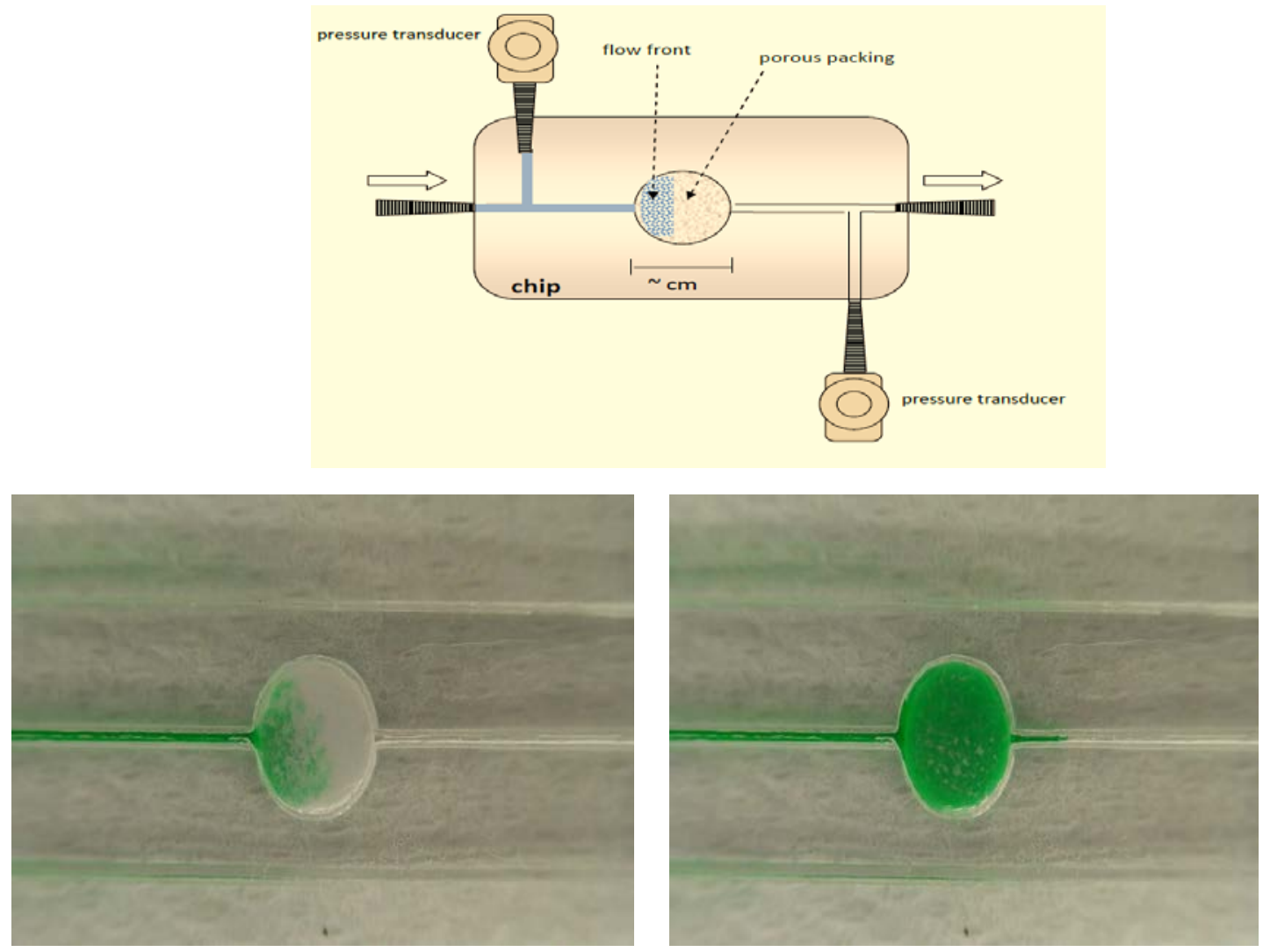

Figure 6: Imaging of flow through a porous filter on a microfluidic chip.
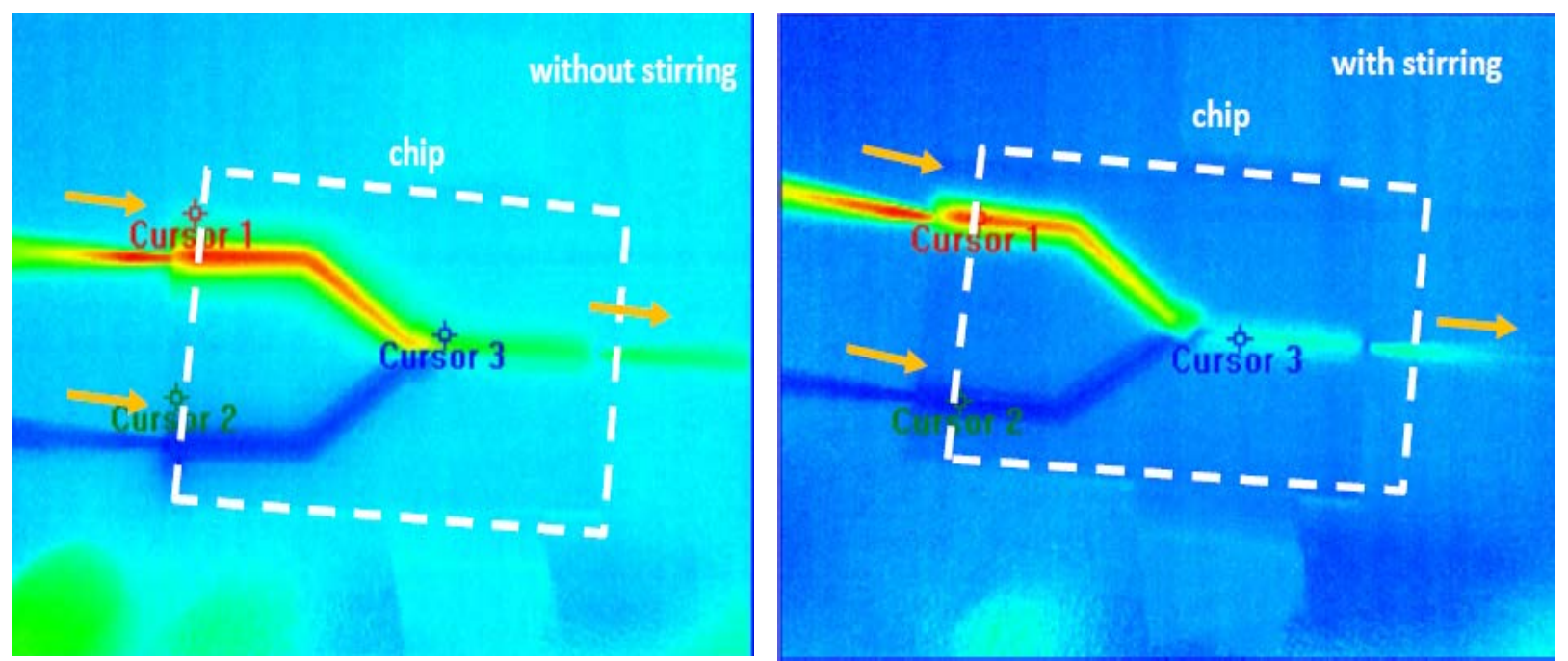

Figure 7: Thermal imaging of fluid flow in microfluidic channels (for microfluidic chip, see Figure 5) Sulfuric acid and $\mathrm{NaOH}$ are combined at a junction, without (left) and with (right) active mixing with a magnetic stir bar. The resulting temperature distributions can be assessed with analysis of the thermal image. 


\section{Discussion and Conclusions}

Increasingly, materials, components, devices, systems, and processes are monitored and interrogated with imaging devices. Images (on a pixel basis) provide thousands - if not millions - of times more data than the point sensors they replace. Imaging data can be made quantitative and much more informative than discrete sensors. Further, most engineering modeling (finite difference, finite element, e.g., COMSOL) produces two- and three-dimensional distributions of temperature, pressure, stress, composition, flow, voltage, etc... Accordingly, imaging can be used to validate such models.

We have successfully incorporated imaging-based laboratories in several undergraduate courses as discussed above (solar cell characterization; and microscale fluid flow, reaction kinetics, and heat transfer). These are comparatively inexpensive experiments that can be done on a desktop with widely available imaging cameras. They can provide instructive examples to students of how imaging can provide vastly more data and information than with traditional point sensors, provide a global assessment, and often are directly interpretable through finite element modeling software.

The availability of low-cost imaging devices will transform healthcare, environmental monitoring, many manufacturing processes, quality assurance, supply chain management, and energy conversion. Imaging schemes that enable quantitative assessment of optical absorption, fluorescence, luminescence, and colorimetry add new dimensions to measurement science, particularly with regard to specific detection and assay of material components such as metal ions, nanoparticles, proteins, DNA, and inorganic species. Further, imaging can be used to evaluate surface roughness and contamination and detect defects, and also map flow patterns in fluid systems. Thermal imaging, increasingly available through cellphone camera attachments, vastly expands the capabilities of temperature measurement. Cellphone platforms, low-cost CCD and infrared cameras, desktop scanners, and other consumer devices, in combination with 3d printing, microcontrollers (e.g., Arduino), and a host of microcontroller-ready sensors and accessories, are a versatile toolbox for sensing, control, computation, and communication. Further, there is much user-friendly image processing freeware (Image ${ }^{\mathrm{TM}}$ ), web-based software $\left(\right.$ Colorpicker $^{\mathrm{TM}}$ ) or image processing utilities bundled with commonly available software (MATLAB $^{\circledR}$ ) for image analysis with varying levels of sophistication. Utilization of image capture technology is thus easily incorporated into educational endeavors. With reference to a low-cost camera phone based spectrometer that he developed, University of Illinois professor Alexander Scheeline commented that "the potential here is to make analytical chemistry for the masses, rather than something done by specialists. There is no doubt getting the cost down to a point where more people can afford them In the education system is a boon for everybody” [35].

The approaches reviewed here suggest many biomedical applications, and serve as an effective means for introducing students to healthcare applications of engineering. For example, lateral flow strips, such as the home pregnancy tests, drugs of abuse testing, and food contamination tests, can be purchased in pharmacies for $\sim \$ 10$. These strips can be imaged with a 
CCD camera to make the test semi-quantitative. Similarly, solutions of DNA and other biomolecules can be quantified by adding fluorescent dyes and measuring fluorescence levels against calibration standards. These serve as two simple examples of introducing engineering students to bioassays. Biosensors and microfluidic based devices will more and more be interconnected via wireless technology to The Internet of Medical Things (IoMT), which is an emerging, highly-connected healthcare network between patients and providers that will improve the quality of healthcare, facilitate more individualized or personalized therapies, lower costs, and expand accessibility, especially in the developing world. Many of the nodes of the IoMT require imaging devices for sensing, patient monitoring, surveillance, and diagnostics. As healthcare comprises an increasingly large ( 20\%) share of the economy, opportunities fostered by low-cost, pervasive imaging for the IoMT and other biomedical applications, along with environmental monitoring, the technology and should be more prominent in the undergraduate engineering curriculum, either integrated into current course laboratories, such as fluid mechanics, heat transfer, and electronics, or as special courses focusing on measurement science with emphasis on image-based data, or in capstone Senior Design Projects.

\section{Acknowledgement}

The authors of this paper would like to thank the Department of Education (Grant No: Award P031S120131) for its financial support towards the project.

\section{References}

1. F Ahmed, "Smartphone spectroscopy takes the lab to the people” Biophotonics (October 2018). https://www.photonics.com/Article.aspx?AID=63917.

2. A Ozcan, "Mobile phones democratize and cultivate next-generation imaging, diagnostics and measurement tools” Lab Chip (2004) 14 3187-3194.

3. D Quesada-González and A Merkoçi, "Mobile phone-based biosensing: An emerging ‘diagnostic and communication' technology” Biosensors Bioelectronics (2017) 92 549562.

4. FM Walker, KM Ahmad, M Eisenstein, and HT Soh, “Transformation of personal computers and mobile phones into genetic diagnostic systems” Analytical Chemistry (2014) 86 9236-9241.

5. DM Scott and H McCann, eds., Process Imaging for Automatic Control (Taylor \& Francis, 2005).

6. AJS McGonigle, TC Wilkes, TD Pering, JR Willmont, JM Cook, FM Mims III, and AV Parisi, "Smartphone spectrometers” Sensors (2018) 18223.

7. AR Campos. CM Knutson, TR Knutson, AR Mozetti, CL Haynes and RL Penn, "Quantifying gold nanoparticle concentration in a dietary supplement using smartphone colorimetry and Google applications” J Chemical Education (2016) 93 318-321. 
8. E Sharpe and S Andreescu, "Integration of nanoparticle-based paper sensors into the classroom: An example of application of rapid colorimetric analysis of antioxidants” $J$. Chemical Education (2015) 92 886-891.

9. AW Martinez, ST Phillips, E Carriho, SW Thomas, H Sindi, and GM Whitesides, "Simple telemedicine for developing regions: Camera phones and paper-based microfluidic devices for real-time, off-site diagnosis” Analytical Chemistry (2018) 80 3699-3707.

10. Li Shen, JA Hagen, and I Papautsky, "Point-of-care colorimetric detection with a smartphone” Lab Chip (2012) 12 4240-4243.

11. Y Lu, W Shi, J Qin, and B Lin, “Low-cost portable detection of gold nanoparticle-labeled microfluidic immunoassay with camera cell phone” Electrophoresis (2009) 30 579-582.

12. GJ Lumetta and E Arcia, "Investigating dissolution and precipitation phenomena with a Smartphone microscope” J Chemical Education (2016) 93, 10 1754-1759.

13. AJ Das, A Wahi, I Kothari and R Raskar, "Ultra-portable, wireless smartphone for rapid, non-destructive testing of fruit ripeness” Scientific Reports (2016) 6:32504.

14. Z Göröcs and A Ozcan, "Biomedical imaging and sensing using flatbed scanners” Lab Chip (2014) 14(17) 3248-3257.

15. YL Shishkin, SG Dmitrienko, OM Medvedeva, SA Badakova, and LN Pyatkova, "Use of a scanner and digital image-processing software for the quantification of adsorbed substances” J Analytical Chemistry (2004) 59, 2 102-106.

16. AG Zheleznyak and VG Sidorov [2105], "Flatbed scanner as an instrument for physical studies” St Petersburg Polytechnical University Journal: Physics and Mathematics 1 134-141.

17. DJ Soldat, P Barak, and BJ Lepore, "Microscale colorimetric analysis using a desktop scanner and automated digital image analysis” J Chemical Education (2009) 86, 5 617620.

18. JA Poce-Fatou, M Bethencourt, FJ Moreno-Dorado, and JM Palacios-Santander, "Using a flatbed scanner to measure detergency: A cost-effective undergraduate laboratory” $J$ Chemical Education (2011) 88 1314-1317.

19. KR Mathews, JD Landmark, and DF Stickle, "Quantitative assay for starch by colorimetry using a desktop scanner” J Chemical Education (2004) 81, 5 702-704. 
20. KV Oskolok, EV Shults, OV Monogarova, and AA Chaplenko, "Optical molecular analysis using office flatbed photo scanner: New approaches and solutions" Talanta (2018) 178 377-383.

21. J Kearns and J Tyson, "Improving the accuracy and precision of an arsenic field test kit: increased reaction time and digital image analysis" Analytical Methods (2012) 4 16931698.

22. MG Michael G. MAuK and Richard Y. Chiou, "Image Capture, Processing and Analysis of Solar Cells for Engineering Education” ASEE Annual Conference \& Exposition Proc. (2015).

23. J-H Jean, C-H Chen, and H-L Lin, "Application of an image processing software tool to crack inspection of crystalline silicon solar cells" Proc 2011 International Conf on Machine Learning and Cybernetics, Guilin (2013) IEEE Press, 1666-1671.

24. Y Wang, P Zhang, S Wang, J Xiong, A Cai, P Huang, and R Sun, “The application of infrared image technology in the detection of local defects of solar cell module" Advanced Materials Research (2012) 347-353 1206-1209.

25. F Luk, V Huynh, and W North, "Measurement of surface roughness by a machine vision system” J Phys E Scientific Instuments (1989) 22 977-980.

26. Z-g Dong, U-j Deng, and Y-z Li, "Surface roughness measurement based on image comparison” 2010 International Conf on Computer, Mechatronics, Control, and Electronic Engineering (CMCE) (2010) IEEE 361-365.

27. T Jeyapoovan and M Murugan, "Surface roughness classification using image processing” Measurement (2013) 46 2064-2072.

28. G Lamour,A Hamraoui, A Buvailo, Y Xing, V Prakash, A Effekhari-Bafrooei, and E Bourget, "Contact angle measurements using a simplified experimental setup" $J$ Chemical Education (2010) 87121403.

29. JB Lee, HR Gwon, SH Lee, and M Cho, "Wetting transition characteristics on microstructured hydrophobic surfaces” Materials Transactions (2010) 51, 9 1709-1711.

30. D Williams, AT Kuhn, TM O’Bryon, and MM Konarik. [2010] “Contact angle measurements using cellphone cameras to implement the Bikerman method" Galvanotechnik (2011) 102, 8 1718-1725.

31. C Xie and E Hazzard, "Infrared imaging for inquiry-based learning” The Physics Teacher (2011) 49, 6 368-372.

32. C Xie [2011], "Visualizing chemistry with infrared imaging” J Chemical Education (2011) 88 881-885. 
33. C Xie, “Transforming science education with IR imaging” InfraMation (2012) paper 67.

34. M Vollmer and K-P Möllmer, Infrared Thermal Imaging: Fundamentals, Research, and Applications, $2^{\text {nd }}$ ed. (Wiley, 2018).

35. T Carmody, Wired: Gadgetlab (http://www.wired.com/gadgetlab/2010. (downloaded 25 Jan 2018). 